\title{
Gait analysis of patients with an off-the-shelf total knee replacement versus customized bi-compartmental knee replacement
}

\author{
Henry Wang ${ }^{1} \cdot$ Jonathan Foster $^{1} \cdot$ Natasha Franksen $^{1} \cdot$ Jill Estes $^{1} \cdot$ Lindsey Rolston ${ }^{2}$
}

Received: 28 January 2017 / Accepted: 15 August 2017 /Published online: 4 September 2017

(C) The Author(s) 2017. This article is an open access publication

\begin{abstract}
Introduction Newer TKR designs have been introduced to the market with the aim of overcoming common sizing problems with older TKR designs. Furthermore, since a sizable percentage of patients with OA present with disease limited to the medial/ lateral knee compartment in addition to the patellofemoral joint, for whom, a customized bi-compartmental knee replacement (BKR) is available as a treatment option. To date, there is very little information regarding knee strength and mechanics during gait for patients implanted with these modern TKR and BKR designs.

The purpose of the study was to evaluate knee strength and mechanics during walking for patients with either a modern off the shelf TKR or a customized BKR and compare these findings to a cohort of healthy controls.

Methods Twelve healthy controls, eight BKR, and nine TKR patients participated in the study. Maximal isometric knee strength was evaluated. 3D kinematic and kinetic analyses were conducted for level walking.

Results The TKR knee exhibited less peak extensor torque when compared to, both the BKR and control limbs $(p<0.05)$. The TKR knee had less extensor moment at stance than both the BKR and control knees $(p<0.05)$. Both the $\mathrm{BKR}$ and control knees displayed larger internal rotation at stance than that of the TKR knee $(p<0.05)$.

Discussion and conclusion This study suggests that, for patients that exhibit isolated OA of the tibiofemoral joint,
\end{abstract}

Henry Wang

hwang2@bsu.edu

1 Biomechanics Laboratory, School of Kinesiology, Ball State University, Muncie, IN 47306, USA

2 Henry County Center for Orthopedics, New Castle, IN 47362, USA using a customized BKR implant is a viable treatment option and may contribute to superior mechanical advantages.

Keywords Knee OA $\cdot$ TKR $\cdot$ BKR $\cdot$ Walking $\cdot$ Knee mechanics

\section{Introduction}

End-stage knee osteoarthritis (OA) is typically treated with total knee replacement (TKR) procedures. One common problem associated with TKRs is reduced knee strength even after multiple years post-surgery [1]. Traditionally, TKR products have been available in a limited number of sizes, which has led to a debate on poor patient outcomes where implant sizing is a contributing factor. Oversized/undersized TKRs can cause patients discomfort during daily activities. Oversized TKRs could result in soft tissue impingement and knee pain [2-4]. Undersized TKRs could lead to joint laxity and instability during weight-bearing activities [5]. Thus, patients with oversized/undersized TKRs may hesitate to exert full effort during knee exercises and weight-bearing activities, which could negatively affect the pace of post-operative recovery. New TKR designs (Persona ${ }^{\mathrm{TM}}$, Zimmer Inc. Warsaw, IN) are now available that aim to address the sizing problem by increasing the number of available sizes [5]. The femoral components are sized in $2 \mathrm{~mm}$ increments in the anteroposterior direction and have standard or narrow mediolateral options [5]. The asymmetric tibial component aims to eliminate the compromise between rotation and fit. These improvements could result in an improved feel and function for patients while performing weight-bearing activities such as walking. To date, it is not known how much of an improvement in knee strength and function patients could gain after implantation with this design. 
End-stage OA typically affects knees in a non-uniform manner with the medial compartment being most affected [6]. The three most common areas affected by OA are the medial compartment, patellofemoral compartment, and medial and patellofemoral compartment overlap [7]. Over a quarter of the patients treated with TKR have OA limited to either the medial or lateral compartment overlapped with the patellofemoral compartment [8]. Thus, there is scope for a bi-compartmental knee replacement that replaces only the affected areas and retains healthy tissues, especially if patients have a competent ACL. Research has shown that previously available monolithic bi-compartmental knee replacements (BKR) replacing the medial and patellofemoral compartments help patients regain normal walking speed with good frontal plane knee mechanics [9]. However, they were shown to have an unsatisfactory safety profile and were withdrawn from the market. An individualized BKR (iDuo ${ }^{\mathrm{TM}}$, ConforMIS Inc. Bedford, MA) utilizes CT scans to make the knee components match the individual's anatomy. The iDuo replaces either the OA-affected medial or lateral compartment and the patellofemoral compartment while retaining the unaffected compartment and cruciate ligaments. It is theorized that the iDuo may produce normal knee mechanics since it replicates an individual's anatomy. To date, it is unclear whether this BKR could facilitate knee strength recovery and enable normal knee mechanics during weightbearing activities.

This study examined knee strength and mechanics during walking in patients who have either a Persona TKR or an iDuo BKR, as compared to healthy controls. As the Persona aims to improve fit of the implant component, TKR patients may exhibit improvements in knee strength and knee mechanics during walking. Similarly, as the iDuo is designed to match each individual's anatomy, BKR patients could experience a return to normal knee strength and exhibit natural knee mechanics during walking. It is hypothesized that the BKR may show greater improvement in knee strength and mechanics than the TKR system due to the retention of more healthy bone, soft tissues, and maintaining patients' knee geometry.

\section{Materials and methods}

Twelve healthy middle-age participants formed a control group (six males, six females). Eight participants (two males, six females) with end-stage $\mathrm{OA}$ in the medial/lateral and patellofemoral compartments treated with the iDuo formed the BKR group. Nine participants (two males, seven females) with end-stage OA treated with the Persona formed the TKR group. A standard parapatellar approach with an anteromedial arthrotomy was used during the knee replacement surgeries, which were conducted by the same surgeon. Standard procedures were followed to perform soft tissue balancing and to correct frontal plane deformity to achieve good knee alignment [10]. All patients received the same rehabilitation program post-surgery.

The BKR group consisted of two bilateral BKR participants and six unilateral BKR participants. The TKR group consisted of three bilateral TKR participants and six unilateral TKR participants. The means and SDs of age, body mass, and height of all patients are reported in Table 1. In addition, postoperative time of the BKR and TKR limbs is presented in Table 2. The study was approved by our institution's Institutional Review Board prior to testing and all patients signed an informed consent prior to enrollment.

Three-dimensional (3D) biomechanical analyses were conducted while participants performed five trials of level walking at a self-selected pace. Ten VICON ${ }^{\mathrm{TM}} \mathrm{F} 40$ cameras capturing data at $100 \mathrm{~Hz}$ (Vicon, Denver, CO) were used to determine the spatial locations of reflective markers on participants. A modified plug-in gait model with additional thigh and shank clusters was used for the placement of the markers on the body. A VICON Nexus ${ }^{\mathrm{TM}}$ system (V 1.8) was used to generate $3 \mathrm{D}$ coordinates of the reflective markers. Three AMTITM force platforms (Model OR6-7, Advanced Mechanical Technology Inc., Watertown, MA) were used to collect ground reaction forces at $2000 \mathrm{~Hz}$. Visual 3D (V 5.1) (C Motion Inc. Germantown, MD) was used to perform link model based computations. A CYBEX NORM isokinetic dynamometer (CSMI, Stoughton, MA) was used to determine maximal isometric knee extensor strength at $60^{\circ}$ of flexion and knee flexor strength at $30^{\circ}$ of flexion. Using these inputs, the following dependent variables were analyzed: maximal isometric knee extensor and flexor torques, peak knee moments and peak knee angles during stance phase of walking, walking speed, and time for double limb support during walking. Knee moments were normalized to body mass in order to compare the results of the three groups. Lastly, scores of the knee outcome survey activities of daily living (KOS-ADL) of the BKR and TKR patients were assessed at the time of testing (Table 2). The KOS-ADL is a patient-reported instrument for the measurement of symptoms and functional limitations that may occur due to pathological disorders and impairments of the knee. The symptoms included in the KOS-ADL are pain, stiffness, swelling, instability, and weakness. The functional limitations included in the KOS-ADL are difficulty of walking on level surfaces and stairs, standing, kneeling, and squatting, sitting, and rising from a sitting position [11].

Statistical analyses were performed using SPSS software (V. 19.0 for Windows, IBM, Inc., Chicago). One-way analysis of variance (ANOVA) was used to determine statistically significant differences among the three groups. Significance level was set at 0.05 . 
Table 1 Means and SDs of age, body mass, body height, body mass index (BMI), double support time, and walking speed of the BKR, TKR, and control group

\begin{tabular}{lccc}
\hline & BKR $(n=8)$ & TKR $(n=9)$ & Control $(n=12)$ \\
\hline Age (yrs.) & $63(10)$ & $65(9)^{\#}$ & $57(6)$ \\
Body mass (kg) & $87(20)$ & $90(35)$ & $82(11)$ \\
Body height $(\mathrm{cm})$ & $166(8)$ & $169(12)$ & $175(11)$ \\
BMI $\left(\mathrm{kg} / \mathrm{m}^{2}\right)$ & $32(8)^{* *}$ & $31(7)^{\#}$ & $27(2)$ \\
Walking speed (m/s) & $1.38(0.12)^{*}$ & $1.19(0.24)^{\#}$ & $1.48(0.17)$ \\
Double support time (\% Gait Cycle) & $24(4)^{*}$ & $34(13)^{\#}$ & $25(4)$ \\
\hline
\end{tabular}

Note. ${ }^{*}$ indicates significant difference $(p<0.05)$ between the BKR and TKR groups,

${ }^{* *}$ indicates significant difference $(p<0.05)$ between the BKR and the control groups, ${ }^{*}$ indicates significant difference $(p<0.05)$ between the TKR and the control groups

\section{Results}

No differences were found among the groups in terms of mass and height $(p>0.05)$. However, the BKR $(p=0.04)$ and TKR groups $(p=0.05)$ had greater body mass index (BMI) than the control group. There were no differences in age between the BKR and TKR groups $(p=0.62)$ or between the BKR and control groups $(p=0.09)$. However, the TKR group was older than the control group $(p=0.02)$ (Table 1). In addition, no difference in post-operative time was found between the BKR and TKR limbs ( $p=0.35$ ) (Table 2). Lastly, the post-operative KOS-ADL scores of the BKR limbs were higher than those of the TKR limbs $(p=0.01)$ (Table 2).

The TKR group walked slower than the BKR $(p=0.02)$ and control groups $(p=0.01)$ (Table 1$)$. No difference in speed was found between the BKR and control group $(p=0.14)$. The TKR group had greater double-support time than the $\operatorname{BKR}(p=0.01)$ and control groups $(p=0.01)$ during walking. There was no difference in double-support time between the BKR and control group $(p=0.68)$.

Kinetic analysis showed that control limbs exerted greater extensor torque than TKR limbs $(p=0.01)$. BKR limbs trended toward greater extensor torque than TKR limbs $(p=0.07)$. No difference in extensor torque was found between the BKR and control limbs $(p=0.14)$ (Table 2). Control limbs also exerted greater flexor torque than both BKR and TKR limbs $(p=0.01)$. No difference in flexor torque was found between the BKR and TKR limbs $(p=0.26)$.
TKR patients exerted less peak extensor moment than both $\operatorname{BKR}(p=0.03)$ and control limbs $(p=0.01)$ (Table 3$)$. No difference in peak extensor moment was observed between the BKR and control limbs $(p=0.06)$. No difference in peak abduction moment during loading response phase was found among the three groups $(p>0.05)$. TKR limbs exhibited less peak abduction moment during push-off than both BKR $(p=0.05)$ and control limbs $(p=0.01)$. There was no difference in peak abduction at stance among the three limbs $(p>0.05)$. Finally, both the BKR $(p=0.03)$ and control limbs $(p=0.01)$ exhibited greater peak internal rotation than the TKR limbs. No difference in peak internal rotation was observed between the BKR and control limbs $(p=0.68)$.

\section{Discussion}

This study evaluated knee strength and mechanics during walking in patients with either a BKR or TKR in comparison to healthy control subjects. During strength testing, both TKR and BKR patients were able to exert maximal contractions with their knee extensors and flexors. However, TKR extensor strength was $34 \%$ less than the control knees. The BKR extensor strength was not significantly different than the control group and was $20 \%$ greater than the TKR group. The significant improvement of the BKR extensor strength may result from the individualized nature of the BKR design and procedure, which removes less bone and soft tissue. In a little over a
Table 2 Means and SDs of postoperative time, scores of knee outcome survey activities of daily living (KOS-ADL), maximal isometric knee extensor, and flexor torques of the BKR, TKR, and control limbs

\begin{tabular}{lccc}
\hline & $\begin{array}{l}\text { BKR limbs } \\
(n=10)\end{array}$ & $\begin{array}{l}\text { TKR limbs } \\
(n=12)\end{array}$ & $\begin{array}{l}\text { Control limbs } \\
(n=12)\end{array}$ \\
\hline Post-operative time (mo.) & $17(9)$ & $14(5)$ & $\mathrm{NA}$ \\
KOS-ADL (\%) & $88(6)^{*}$ & $75(16)$ & $\mathrm{NA}$ \\
Max isometric knee extensor torque $(\mathrm{Nm} / \mathrm{kg})$ & $1.65(0.53)$ & $1.37(0.33)^{\#}$ & $2.07(0.71)$ \\
Max isometric knee flexor torque $(\mathrm{Nm} / \mathrm{kg})$ & $0.74(0.20)^{* *}$ & $0.79(0.20)^{\#}$ & $1.10(0.31)$ \\
\hline${ }^{* *}{ }^{*}$ indicates significant difference $(p<0.05)$ between the BKR and TKR limbs, & \\
indicates significant difference $(p<0.05)$ between the BKR and the control limbs, ${ }^{*}$ indicates significant \\
difference $(p<0.05)$ between the TKR and the control limbs
\end{tabular}


Table 3 Means and SDs of typical variables of knee mechanics at stance (peak knee extensor moment, peak knee abduction moments during loading response and push-off, peak knee abduction, peak knee internal rotation) of the BKR, TKR, and control limbs

\begin{tabular}{lllc}
\hline Variables of knee mechanics & $\begin{array}{l}\text { BKR limbs } \\
(n=10)\end{array}$ & $\begin{array}{l}\text { TKR limbs } \\
(n=12)\end{array}$ & $\begin{array}{l}\text { Control limbs } \\
(n=12)\end{array}$ \\
\hline Peak knee extensor moment $(\mathrm{Nm} / \mathrm{kg})$ & $0.63(0.20)^{*}$ & $0.48(0.16)^{\#}$ & $0.81(0.23)$ \\
Peak knee abduction moment during loading response $(\mathrm{Nm} / \mathrm{kg})$ & $0.41(0.12)$ & $0.40(0.07)$ & $0.40(0.11)$ \\
Peak knee abduction moment during push-off $(\mathrm{Nm} / \mathrm{kg})$ & $0.23(0.06)^{*}$ & $0.19(0.06)^{\#}$ & $0.27(0.08)$ \\
Peak knee abduction $\left(^{\circ}\right)$ & $7(5)$ & $7(2)$ & $5(3)$ \\
Peak knee internal rotation $\left(^{\circ}\right)$ & $6(5)^{*}$ & $3(3)^{\#}$ & $7(3)$ \\
\hline
\end{tabular}

Note. ${ }^{*}$ indicates significant difference $(p<0.05)$ between the BKR and TKR limbs,

*** indicates significant difference $(p<0.05)$ between the BKR and the control limbs, ${ }^{\#}$ indicates significant difference $(p<0.05)$ between the TKR and the control limbs year, the BKR patients were able to elevate their extensor strength to a level similar to the control population. Unfortunately, both BKR and TKR limbs showed weaker knee flexors than the control with a strength deficit of $32 \%$ and $28 \%$, respectively, possibly due to both groups having an average BMI $>30$. None of the patients regularly participated in fitness or strength training programs focusing on the knee after the standard rehabilitation. Strength may be improved for both implant groups if patients participate in regular fitness programs and strength training within one year of surgery. Nonetheless, both the BKR and TKR patient groups reported good KOS-ADL scores at the time of testing. The higher KOS-ADL scores in BKR patients may be a result of the significant improvement of the BKR extensor strength when compared to the TKR patients.

During walking, TKR patients exhibited weakness in the sagittal plane. On average, the TKR group generated $40 \%$ and $24 \%$ less extensor moment than the control and BKR groups, respectively. The significant inability of the TKR knees to produce adequate extensor moment during walking is associated with the knee strength deficit. Due to the decreased extensor moment, TKR patients experienced increased duration of double limb support that was $36 \%$ and $41 \%$ longer than the control and BKR patients, respectively, during stance phase of gait. With smaller knee extensor moment and longer double-support time, TKR patients demonstrated a $20 \%$ and a $14 \%$ slower walking pace than the control and BKR patients, respectively. Although it is not uncommon to see knee replacement patients walking at a reduced speed with decreased knee extensor moment after one year of surgery, the BKR patients were able to improve their knee extensor moment and walking speed to a level similar to the controls. The advantages associated with the BKR, such as the individualized nature of the implant, small bone cuts, and reduced soft tissue intervention, may have contributed to these significant improvements.

Both the BKR and TKR knees were found to be well aligned. During stance of walking, the peak abduction angles of the BKR and TKR knees were as small as that of the control knees. It is also encouraging to see that during the loading response phase of walking, the peak abduction moment of both implant groups were close to that of the controls. With small peak abduction angle and peak abduction moment, the artificial knees in this study are expected to experience reduced mechanical stress in the medial side of the knees during daily walking, which could lead to increased implant survivorship.

During push-off, TKR knees exhibited a significant reduction of the peak abduction moment when compared to the BKR and controls limbs. This pattern may be related to the strength deficit of the TKR knees. It is possible that the reduced extensor moment during stance led to a reduction in medial knee loading signified by the decreased knee abduction moment. However, both the BKR and control knees produced similar amount of abduction moment at push-off, which corresponded to the similar extensor moment between the two knees.

It has been well documented that normal knees exhibit internal rotation of the tibia during flexion $[12,13]$. We observed that the control knees displayed normal tibial rotation, which was represented by a $7^{\circ}$ internal rotation during the stance phase of walking. When kinematics of the knee replacements were examined, the BKR knees produced a comparable amount of internal rotation $\left(6^{\circ}\right)$ to that of the controls. The TKR knees also displayed some internal rotation $\left(3^{\circ}\right)$, but the range of the rotation was limited and was $50 \%$ less than that of the BKR and control limbs. Ideally, knee replacement systems should help patients obtain natural knee kinematics during weight-bearing activities. Natural knee kinematics could promote a natural feel for patients while going through rehabilitation programs and may accelerate the recovery of knee strength and function. BKR patients demonstrated comparable knee kinematics as assessed from knee rotation to the controls as well as satisfactory knee strength at one year post-surgery. A recent study showed that customized TKRs manufactured using the same $\mathrm{CT}$ image to implant process are better suited to replicate normal knee kinematics when compared to non-customized TKR [14]. Thus, the individualized shape of the customized implant may play an important role in improving kinematic function. 
As with any study, there are some limitations that need consideration when interpreting our results. The sample size of participants in each group was smaller than the typical follow-up studies that report on functional and clinical endpoints. Though sample size plays an important role in interpreting results, we believe from our prior experience with conducting such studies [9,15-18], that the sample size chosen was adequate to enable us to make conclusions on our analyses. Additionally, we were able to maintain a similar sample size in each arm of the study. This should alleviate any bias due to sample size in any one study arm. Although participants in the control group were younger with smaller BMI than the other groups, the two patient groups were age, mass, and height matched. We believe that any advantage drawn from this would affect the implant groups equally, thus making comparisons between the implant groups relevant, while still providing context on how they compare to healthy controls. Ideally, we would have liked to test patients pre- and post-operatively and compare results with the patient being their own control. However, this would mean having to test patients that have end-stage OA, which we felt would not provide a clear comparison to healthy controls. Lastly, in this study, patients' pre-operative Knee Society scores and gait analysis data were not available due to our cross-sectional study design. However, we believe our patients' pre-surgical conditions were similar to patients used in other prospective studies examining functional improvements after knee replacements. In those studies, patients' combined Knee Society scores were close to 100 and knee range of motion was around $120^{\circ}$ [19-22]. In general, patients with end-stage knee OA experience joint pain and stiffness, which leads to functional limitations of performing daily activities such as walking, going up and down stairs, and rising from a sitting position. We chose the KOS-ADL because it is an effective instrument for measuring functional limitations associated with pathological disorders of the knee [11]. However, we only administered the KOS-ADL during patients' postoperative laboratory visit. Ideally, if the KOS-ADL score was obtained prior to surgery, then it is possible to quantify how much functional improvement is made at the time of the post-operative laboratory testing.

In summary, after one year post-surgery, both patient groups had good KOS-ADL scores with the BKR patients reporting higher satisfaction while performing activities of daily living. Patients with BKR knees were able to improve their quadriceps strength to a level that is close to healthy controls. BKR patients could walk at a speed similar to the controls with adequate extensor moment. BKR knees also revealed good knee mechanics represented by a small abduction angle, normal abduction moment, and normal internal rotation, which were comparable to those of healthy controls. The TKR knees had deficits in strength that led to a reduced walking speed and decreased extensor moment. Some improvements in knee mechanics were shown by the TKR limbs. Similar to the other groups, the TKR knees displayed a small abduction and normal abduction moment. Improved results seen in the BKR patients can be attributed to the ability of a BKR surgery to maintain both cruciate ligaments as well as the customized nature of the implant facilitating a more normal kinematic pattern. As the aging population increases in the US, it is expected that approximately 3.48 million patients will receive TKRs in 2030 [23]. Studies have shown that nearly $30 \%$ of TKR recipients exhibit OA limited in the medial/lateral compartment and the patellofemoral joint [8]. These patients benefit from BKRs, as this study shows that BKR patients exhibit better strength and mechanics while performing activities of daily living. Patients with modern TKR systems such as the one examined in this study may consider participating in strength training programs for further improvement in knee strength and function.

Funding ConforMIS Inc.

\section{Compliance with ethical standards}

Conflict of interest Author HW received a research grant from ConforMIS Inc. All authors declare that there is no conflict of interest.

Ethical approval All procedures performed in studies involving human participants were in accordance with the ethical standards of the institutional and/or national research committee and with the 1964 Helsinki declaration and its later amendments or comparable ethical standards.

Informed consent Informed consent was obtained from all individual participants included in the study.

Open Access This article is distributed under the terms of the Creative Commons Attribution 4.0 International License (http:// creativecommons.org/licenses/by/4.0/), which permits unrestricted use, distribution, and reproduction in any medium, provided you give appropriate credit to the original author(s) and the source, provide a link to the Creative Commons license, and indicate if changes were made.

\section{References}

1. Huang CH, Cheng CK, Lee YT, Lee KS (1996) Muscle strength after successful total knee replacement, a 6- to 13-year followup. Clin Orthop Relat Res 328:147-154

2. Mahoney OM, Kinsey T (2010) Overhand of the femoral component in total knee arthroplasty: risk factors and clinical consequences. J Bone Joint Surg Am 92-5:1115-1121

3. Petersen W, Rembitzki I, Bruggemann G, Ellermann A, Best R, Gosele-Koppenburg A et al (2014) Anterior knee pain after total knee arthroplasty: a narrative review. Int Orthop 38:319-328

4. Bonnin M, Schmidt A, Basiglini L, Bossard N, Dantony E (2013) Mediolateral oversizing influences pain, function, and flexion after TKA. Knee Surg Sports Traumatol Arthrosc 21:2314-2324

5. Dai Y, Scuderi G, Penninger C, Bischoff J, Rosenberg A (2014) Increased shape and size offerings of femoral components improve fit during total knee arthroplasty. Knee Surg Sports Traumatol Arthrosc 22:2931-2940 
6. Windsor R, Insall J (1994) Surgery of the knee. In: Sledge C, Ruddy S, Harris E, Kelly W (eds) Arthritis surgery. Saunders, Philadelphia, pp 794-817

7. McAlindon TE, Cooper C, Kirwan J, Dieppe P (1992) Knee pain and disability in the community. Br J Rheumatol 31-3:189-192

8. Heekin R, Fokin A (2014) Incidence of bicompartmental osteoarthritis in patients undergoing total and unicompartmental knee arthroplasty: is the time ripe for a less radical treatment? J Knee Surg 27-1:77-81

9. Wang H, Dugan E, Frame J, Rolston L (2009) Gait analysis after bicompartmental knee replacement. Clin Biomech 24:751-754

10. Rolston L, Siewert K (2009) Assessment of knee alignment after bicompartmental knee arthroplasty. J Arthroplast 24-7:1111-1114

11. Irrgang JJ, Snyder-Mackler L, Wainner RS, Fu FH, Harner CD (1998) Development of a patient-reported measure of function of the knee. J Bone Joint Surg Am 80:1132-1145

12. Oatis C (2004) Kinesiology: the mechanics \& pathomechanics of human movement, 1st edn. Lippincott Williams \& Wilkins, Philadelphia

13. Komistek RD, Dennis DA, Mahfouz M (2003) In vivo fluoroscopic analysis of the normal human knee. Clin Orthop Relat Res 410:69-81

14. Zeller I, Sharma A, Kurtz W, Anderle M, Komistek RD (2017) Customized versus patient-sized cruciate-retaining total knee arthroplasty: an in vivo kinematics study using mobile fluoroscopy. J Arthroplast 32-4:1344-1350

15. Wang H, Simpson KJ, Chamnongkich S, Kinsey T, Mahoney OM (2005) A biomechanical comparison between the single-axis and multi-axis total knee arthroplasty systems for the stand-to-sit movement. Clin Biomech 20:428-433
16. Wang H, Simpson KJ, Ferrara MS, Chamnongkich S, Kinsey T, Mahoney OM (2006) Biomechanical differences exhibited during sit-to-stand between total knee arthroplasty designs of varying radii. J Arthroplast 21-8:1193-1199

17. Wang H, Simpson KJ, Chamnongkich S, Kinsey T, Mahoney OM (2008) Biomechanical influence of TKA sesigns with varying radii on bilateral TKA patients during sit-to-stand. Dyn Med 7:12

18. Wang H, Frame J, Rolston L (2012) Influence of bicompartmental knee replacement on stand-to-sit movement. Res Q Exerc Sport 832:136-142

19. Confalonieri N, Manzotti A (2006) Tissue-sparing surgery with the bi-unicompartmental knee prosthesis: retrospective study with minimum follow-up of 36 months. J Orthop Traumatol 7:108-112

20. Bade MJ, Kohrt WM, Stevens-Lapsley JE (2010) Outcomes before and after total knee arthroplasty compared to healthy adults. J Orthop Sports Phys Ther 40:559-567

21. Bolanos AA, Colizza W, McCann PD, Gotlin RS, Wootten ME, Kahn BA, Insall JN (1998) A comparison of isokinetic strength testing and gait analysis in patients with posterior cruciateretaining and substituting knee arthroplasties. J Arthroplast 13: 906-915

22. Kleijn L, van Hemert W, Meijers W, Kester A, Lisowski L, Grimm B, Heyligers I (2007) Functional improvement after unicompartmental knee replacement: a follow-up study with a performance based knee test. Knee Surg Sports Traumatol Arthrosc 15:1187-1193

23. Kurtz S, Ong K, Lau E, Mowat F, Halpern M (2005) Projections of primary and revision hip and knee arthroplasty in the United States from 2005-2030. J Bone Joint Surg Am 89-A4:780-785 